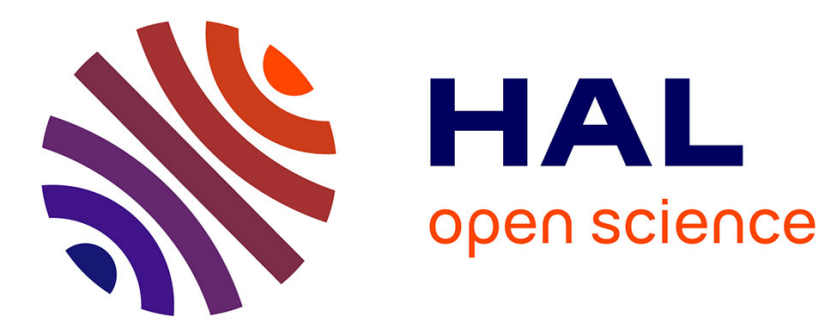

\title{
The mortar spectral element method in domains of operators Part II: The curl operator and the vector potential problem
}

Mjedi Azaïez, Faker Ben Belgacem, Christine Bernardi, Mohammed El Rhabi

\section{- To cite this version:}

Mjedi Azaïez, Faker Ben Belgacem, Christine Bernardi, Mohammed El Rhabi. The mortar spectral element method in domains of operators Part II: The curl operator and the vector potential problem. Annali di Matematica Pura ed Applicata, 2008, 187 (3), pp.405-433. 10.1007/s10231-007-0049-y . hal-00112170

\section{HAL Id: hal-00112170 \\ https://hal.science/hal-00112170}

Submitted on 10 Nov 2006

HAL is a multi-disciplinary open access archive for the deposit and dissemination of scientific research documents, whether they are published or not. The documents may come from teaching and research institutions in France or abroad, or from public or private research centers.
L'archive ouverte pluridisciplinaire HAL, est destinée au dépôt et à la diffusion de documents scientifiques de niveau recherche, publiés ou non, émanant des établissements d'enseignement et de recherche français ou étrangers, des laboratoires publics ou privés. 


\title{
The mortar spectral element method in domains of operators
}

\section{Part II: The curl operator and the vector potential problem}

\author{
by M. Azaïez ${ }^{1}$, F. Ben Belgacem ${ }^{2}$, C. Bernardi ${ }^{3}$ and M. El Rhabi ${ }^{4}$
}

\begin{abstract}
The mortar spectral element method is a domain decomposition technique that allows for discretizing second- or fourth-order elliptic equations when set in standard Sobolev spaces. The aim of this paper is to extend this method to problems formulated in the space of square-integrable vector fields with square-integrable curl. We consider the problem of computing the vector potential associated with a divergence-free function in dimension 3 and propose a discretization of it. The numerical analysis of the discrete problem is performed and numerical experiments are presented, they turn out to be in good coherency with the theoretical results.
\end{abstract}

Résumé: La méthode d'éléments spectraux avec joints est une technique de décomposition de domaine permettant de discrétiser des équations elliptiques d'ordre 2 ou 4 posés dans des espaces de Sobolev usuels. Le but de cet article est d'étendre cette méthode à certains problèmes variationnels formulés dans des espaces de champs de vecteurs de carré intégrable à rotationnel de carré intégrable. On considère le problème consistant à calculer le potentiel vecteur associé à une fonction à divergence nulle en dimension 3 et on en propose une discrétisation. On effectue l'analyse numérique du problème discret et on présente des expériences numériques cohérentes avec les résultats de l'analyse.

1 Laboratoire TREFLE (UMR C.N.R.S. 8508), Site E.N.S.C.P.B., 16 avenue Pey Berland, 33607 Pessac Cedex, France. — azaiez@enscpb.fr

2

L.M.A.C. (E.A. 2222), Département de Génie Informatique,

Université de Technologie de Compiègne, Centre de Recherches de Royallieu,

B.P. 20529, 60205 Compiègne Cedex, France. — faker.ben-belgacem@utc.fr

3 Laboratoire Jacques-Louis Lions, C.N.R.S. \& Université Pierre et Marie Curie, B.C. 187, 4 place Jussieu, 75252 Paris Cedex 05, France. - bernardi@ann.jussieu.fr

4 Realeyes3D S.A., 217 Bureaux de la Colline, 92213 Saint-Cloud Cedex, France. — melrhabi@realeyes3d.fr 



\section{Introduction.}

The mortar element method, due to Bernardi, Maday and Patera [6], is a domain decomposition technique which allows for working on general partitions of the domain, without conformity restrictions, see [8] for a recent review. It is specially important when combined with spectral type discretizations, since handling complex geometries from simple subdomains can be performed with this method in a very efficient way. It can also be used to couple different kinds of variational discretizations on the subdomains, such as finite elements or spectral methods. It leads to discrete problems which are most often non conforming in the Hodge sense, which means that the discrete space is not contained in the variational one. It was first analyzed for problems which admit a variational formulation in the usual Sobolev spaces. However a number of interesting problems involve other types of Hilbert spaces which are often domains of operators issued from mechanics and physics. Let us quote among them the spaces $H(\operatorname{div}, \Omega)$ of square-integrable vector fields with square-integrable divergence and the space $H(\operatorname{curl}, \Omega)$ of square-integrable vector fields with square-integrable curl. Up to now, the mortar method has not yet been applied in this case, except when associated with finite element discretizations [3][14][22] and also for Darcy's equations which model the flow in a porous medium and are formulated in $H(\operatorname{div}, \Omega)$, see [2]. We refer to [19] and [10] for first works in the spectral element case concerning Maxwell's system. The aim of this paper is to extend the results of [2] to the space $H(\operatorname{curl}, \Omega)$ in the case of a three-dimensional domain $\Omega$.

The problem that we have chosen to illustrate the interest of the discretization relies on the standard result that a two- or three-dimensional vector field can be written as the sum of a gradient and a curl, the uniqueness of this decomposition being enforced by appropriate gauge and boundary conditions, see [20, Chap. I, Thm 3.2] and [1, §3.e] for a detailed study. Moreover, when the vector field is divergence-free, only the curl part subsists: the vector field is the curl of a scalar function called stream function in the case of dimension 2 , of a vector field called vector potential in the three-dimensional case. In some applications, it could be interesting to compute the curl part of the decomposition. More interesting is the fact that very often a divergence-free vector field is well approximated by a discrete function which has a small but non-zero divergence, and computing the curl part of the decomposition of this approximate function leads to an accurate approximation of the initial vector field which has the further property of being exactly divergence-free. This turns out to be useful for a number of applications (for instance, when the discrete function is involved in a convection equation). It can be noted that a similar system also models stationary magnetic fields $[9][22, \S 2.2 .2]$. So, we propose a mortar spectral element discretization of this computation of the curl part which works even in complex three-dimensional geometries such as multiply-connected ones. We prove a priori error estimates of spectral type, which are optimal for conforming decompositions and nearly optimal otherwise.

A key idea for the implementation of the mortar technique has been introduced in [4], it consists in handling the matching conditions on the interfaces between the elements by introducing a Lagrange multiplier. We present the extension of this new formulation to the type of problems which is analyzed here, next we write the resulting linear system

and we present the algorithm which is used to solve it. Some numerical experiments are described, we check that they are in good agreement with the theoretical results. 
An outline of the paper is as follows.

- In Section 2, we recall the main properties of the space $H(\operatorname{curl}, \Omega)$. We present the vector potential problem and prove its well-posedness.

- Section 3 is devoted to the description of the discretization of this problem and also to the existence of a solution for the discrete problem.

- In Section 4, we derive a priori error estimates for the problem.

- In Section 5, we present some numerical experiments and check the accuracy of the method. 


\section{The space $H(\operatorname{curl}, \Omega)$ and the vector potential problem.}

Let $\Omega$ denote a bounded connected domain in $\mathbb{R}^{3}$, with a Lipschitz-continuous boundary. We denote by $\boldsymbol{n}$ the unit outward normal to $\Omega$ on $\partial \Omega$. The generic point in $\Omega$ is denoted by $\boldsymbol{x}=(x, y, z)$, while the components of any vector field $\boldsymbol{v}$ in $\mathbb{R}^{3}$ are denoted by $v_{x}, v_{y}$ and $v_{z}$.

The curl operator is defined on smooth functions by

$$
\operatorname{curl} \boldsymbol{v}=\left(\begin{array}{l}
\partial_{y} v_{z}-\partial_{z} v_{y} \\
\partial_{z} v_{x}-\partial_{x} v_{z} \\
\partial_{x} v_{y}-\partial_{y} v_{x}
\end{array}\right)
$$

and on all functions $\boldsymbol{v}$ in $L^{2}(\Omega)^{3}$ in the distribution sense:

$$
\begin{aligned}
& \forall \boldsymbol{\varphi} \in \mathscr{D}(\Omega)^{3} \\
& \quad\langle\operatorname{curl} \boldsymbol{v}, \boldsymbol{\varphi}\rangle=\int_{\Omega}\left(v_{x}\left(\partial_{y} \varphi_{z}-\partial_{z} \varphi_{y}\right)+v_{y}\left(\partial_{z} \varphi_{x}-\partial_{x} \varphi_{z}\right)+v_{z}\left(\partial_{x} \varphi_{y}-\partial_{y} \varphi_{x}\right)\right)(\boldsymbol{x}) d \boldsymbol{x} .
\end{aligned}
$$

We introduce the space

$$
H(\operatorname{curl}, \Omega)=\left\{\boldsymbol{v} \in L^{2}(\Omega)^{3} ; \operatorname{curl} \boldsymbol{v} \in L^{2}(\Omega)^{3}\right\}
$$

provided with the norm

$$
\|\boldsymbol{v}\|_{H(\operatorname{curl}, \Omega)}=\left(\|\boldsymbol{v}\|_{L^{2}(\Omega)^{3}}^{2}+\|\operatorname{curl} \boldsymbol{v}\|_{L^{2}(\Omega)^{3}}^{2}\right)^{\frac{1}{2}} .
$$

It is readily checked that $H(\operatorname{curl}, \Omega)$ is a Hilbert space. Moreover, the space $\mathscr{C}^{\infty}(\bar{\Omega})^{3}$ of indefinitely differentiable functions on $\bar{\Omega}$ is dense in $H(\operatorname{curl}, \Omega)$, see [20, Chap. I, Thm 2.10]. This leads to the trace theorem on $H(\operatorname{curl}, \Omega)$.

Proposition 2.1. The trace operator: $\boldsymbol{v} \mapsto \boldsymbol{v} \times \boldsymbol{n}$, defined from the formula

$$
\forall \boldsymbol{\varphi} \in H^{1}(\Omega)^{3}, \quad\langle\boldsymbol{v} \times \boldsymbol{n}, \boldsymbol{\varphi}\rangle=\int_{\Omega}(\boldsymbol{v} \cdot \operatorname{curl} \boldsymbol{\varphi}-\operatorname{curl} \boldsymbol{v} \cdot \boldsymbol{\varphi})(\boldsymbol{x}) d \boldsymbol{x},
$$

is continuous from $H(\mathbf{c u r l}, \Omega)$ into the dual space $H^{-\frac{1}{2}}(\partial \Omega)^{3}$.

Remark: The trace operator is not onto $H^{-\frac{1}{2}}(\partial \Omega)^{3}$ but onto a closed subspace of $H^{-\frac{1}{2}}(\partial \Omega)^{3}$ which has been characterized in [23], see also [12] and [13].

Remark: Let $\Gamma$ be a connected part of $\partial \Omega$ with a positive measure. The trace operator: $\boldsymbol{v} \mapsto \boldsymbol{v} \times \boldsymbol{n}$ is also continuous from $H(\mathbf{c u r l}, \Omega)$ into the dual space $H_{00}^{\frac{1}{2}}(\Gamma)^{\prime 3}$ of $H_{00}^{\frac{1}{2}}(\Gamma)^{3}$, as explained in [11] when $\Omega$ is a polyhedron.

We finally introduce the subspace

$$
H_{0}(\operatorname{curl}, \Omega)=\{\boldsymbol{v} \in H(\operatorname{curl}, \Omega) ; \boldsymbol{v} \times \boldsymbol{n}=0 \text { on } \partial \Omega\}
$$


which is also a Hilbert space. Moreover, the space $\mathscr{D}(\Omega)^{3}$ is dense in $H_{0}(\mathbf{c u r l}, \Omega)$, see [20, Chap. I, Thm 2.12].

The divergence operator in the case of dimension $d=3$ is defined on all smooth vector fields by

$$
\operatorname{div} \boldsymbol{v}=\partial_{x} v_{x}+\partial_{y} v_{y}+\partial_{z} v_{z}
$$

and also on all functions $\boldsymbol{v}$ in $L^{2}(\Omega)^{3}$ in the distribution sense. With this operator, we can associate the space

$$
H(\operatorname{div}, \Omega)=\left\{\boldsymbol{v} \in L^{2}(\Omega)^{3} ; \operatorname{div} \boldsymbol{v} \in L^{2}(\Omega)\right\}
$$

It must be observed that, in contrast with $H(\mathbf{c u r l}, \Omega)$ and $H_{0}(\mathbf{c u r l}, \Omega)$, the intersection $H_{0}(\mathbf{c u r l}, \Omega) \cap H(\operatorname{div}, \Omega)$ has some further regularity properties: It is continuously imbedded in $H^{\frac{1}{2}}(\Omega)^{3}$ [15] and, if the domain $\Omega$ is convex, in $H^{1}(\Omega)^{3}[1$, Thm 2.17]. Further results are known [16][17][18] when $\Omega$ is a polyhedron: A function $\boldsymbol{u}$ in $H_{0}(\mathbf{c u r l}, \Omega) \cap H(\operatorname{div}, \Omega)$, can be written

$$
\boldsymbol{u}=\boldsymbol{u}_{r}+\operatorname{grad} S
$$

where $\boldsymbol{u}_{r}$ belongs to $H^{1}(\Omega)^{3}$ and $S$ is a linear combination of the singularities of the Laplace equation provided with Dirichlet boundary conditions.

We now recall from $[1, \S 3 . e]$ a key result which holds in any bounded connected threedimensional domain $\Omega$ with a Lipschitz-continuous boundary. We introduce some notation concerning the geometry of $\Omega$.

- First, we denote by $\Gamma_{i}, 0 \leq i \leq I$, the connected components of the boundary $\partial \Omega$.

- It is standard to note that there exist $J$ disjoint open cuts $\Sigma_{j}, 1 \leq j \leq J$, which are parts of smooth manifolds, such that each $\partial \Sigma_{j}$ is contained in $\partial \Omega$ and that the open set $\widetilde{\Omega}=\Omega \backslash \cup_{j=1}^{J} \Sigma_{j}$ is simply-connected. We make the further assumption that $\widetilde{\Omega}$ is pseudoLipschitz, in the sense that each point of $\partial \widetilde{\Omega}$ admits a neighbourhood that is made of one or two connected components with Lipschitz-continuous boundaries (see [1] for a more precise definition).

Next, for any vector field $\boldsymbol{u}$ in $L^{2}(\Omega)^{3}$, there exists a function $q$ in $H^{1}(\widetilde{\Omega})$ and a function $\boldsymbol{\psi}$ in $H(\operatorname{curl}, \Omega) \cap H(\operatorname{div}, \Omega)$ such that (here, grad stands for the gradient operator on $\widetilde{\Omega})$

$$
\boldsymbol{u}=\widetilde{\operatorname{grad}} q+\operatorname{curl} \boldsymbol{\psi} \text { in } \Omega,
$$

with the following properties:

$$
\begin{aligned}
& \operatorname{div} \boldsymbol{\psi}=0 \quad \text { in } \Omega, \\
& \boldsymbol{\psi} \times \boldsymbol{n}=\mathbf{0} \quad \text { on } \partial \Omega, \\
& \langle\boldsymbol{\psi} \cdot \boldsymbol{n}, 1\rangle_{\Gamma_{i}}=0, \quad 0 \leq i \leq I .
\end{aligned}
$$

Moreover, let us consider a vector field $\boldsymbol{u}$ in $L^{2}(\Omega)^{3}$ which satisfies

$$
\begin{aligned}
& \operatorname{div} \boldsymbol{u}=0 \quad \text { in } \Omega, \\
& \boldsymbol{u} \cdot \boldsymbol{n}=0 \quad \text { on } \partial \Omega, \\
& \langle\boldsymbol{u} \cdot \boldsymbol{n}, 1\rangle_{\Sigma_{j}}=0, \quad 1 \leq j \leq J .
\end{aligned}
$$


With these assumptions, the function $q$ in (2.6) cancels. So the solution $\boldsymbol{\psi}$ is a solution of the problem

$$
\begin{cases}\operatorname{curl} \boldsymbol{\psi}=\boldsymbol{u} & \text { in } \Omega, \\ \operatorname{div} \boldsymbol{\psi}=0 & \text { in } \Omega, \\ \boldsymbol{\psi} \times \boldsymbol{n}=\mathbf{0} & \text { on } \partial \Omega, \\ \langle\boldsymbol{\psi} \cdot \boldsymbol{n}, 1\rangle_{\Gamma_{i}}=0, & 0 \leq i \leq I .\end{cases}
$$

We now write the variational formulation of problem (2.8). Among the connected components $\Gamma_{i}, 0 \leq i \leq I$, of $\partial \Omega$, we agree to denote by $\Gamma_{0}$ the boundary of the only unbounded connected component of $\mathbb{R}^{3} \backslash \bar{\Omega}$ and we introduce the space

$$
H(\Omega)=\left\{\mu \in H^{1}(\Omega) ; \mu=0 \text { on } \Gamma_{0} \text { and } \mu=\text { constant on } \Gamma_{i}, 1 \leq i \leq I\right\} .
$$

Next, we consider the variational system:

Find $(\boldsymbol{\psi}, \theta)$ in $H_{0}(\mathbf{c u r l}, \Omega) \times H(\Omega)$ such that

$$
\begin{aligned}
& \forall \boldsymbol{\xi} \in H_{0}(\operatorname{curl}, \Omega), \\
& \qquad \int_{\Omega} \operatorname{curl} \psi \cdot \operatorname{curl} \boldsymbol{\xi} d \boldsymbol{x}+\int_{\Omega} \boldsymbol{\xi} \cdot \operatorname{grad} \theta d \boldsymbol{x}=\int_{\Omega} \boldsymbol{u} \cdot \operatorname{curl} \boldsymbol{\xi} d \boldsymbol{x}, \\
& \forall \mu \in H(\Omega), \quad \int_{\Omega} \boldsymbol{\psi} \cdot \operatorname{grad} \mu d \boldsymbol{x}=0 .
\end{aligned}
$$

Indeed, from the density of $\mathscr{D}(\Omega)^{3}$ in $H_{0}(\operatorname{curl}, \Omega)$, it is readily checked that, for any function $\boldsymbol{\psi}$ satisfying $(2.8)$, the pair $(\boldsymbol{\psi}, 0)$ is a solution of (2.10). Conversely, if problem (2.10) admits a solution of the form $(\boldsymbol{\psi}, 0)$, the fnction $\boldsymbol{\psi}$ is a solution of (2.8).

Problem (2.10) is of saddle-point type, so we introduce the kernel

$$
W=\left\{\boldsymbol{\xi} \in H_{0}(\operatorname{curl}, \Omega) ; \forall \mu \in H(\Omega), \int_{\Omega} \boldsymbol{\xi} \cdot \operatorname{grad} \mu d \boldsymbol{x}=0\right\} .
$$

It is readily checked from the definition of $H(\Omega)$ that

$$
W=\left\{\boldsymbol{\xi} \in H_{0}(\operatorname{curl}, \Omega) ; \operatorname{div} \boldsymbol{\xi}=0 \text { in } \Omega \text { and }\langle\boldsymbol{\xi} \cdot \boldsymbol{n}, 1\rangle_{\Gamma_{i}}=0,0 \leq i \leq I\right\} .
$$

We now consider the problem:

Find $\boldsymbol{\psi}$ in $W$ such that

$$
\forall \boldsymbol{\xi} \in W, \quad \int_{\Omega} \operatorname{curl} \psi \cdot \operatorname{curl} \boldsymbol{\xi} d \boldsymbol{x}=\int_{\Omega} \boldsymbol{u} \cdot \operatorname{curl} \boldsymbol{\xi} d \boldsymbol{x}
$$

It is proven in $[1$, Cor. 3.19] that the quantity

$$
\|\operatorname{curl} \boldsymbol{\xi}\|_{L^{2}(\Omega)^{3}}+\|\operatorname{div} \boldsymbol{\xi}\|_{L^{2}(\Omega)}+\sum_{i=0}^{I}\left|\langle\boldsymbol{\xi} \cdot \boldsymbol{n}, 1\rangle_{\Gamma_{i}}\right|,
$$


is a norm on $H_{0}(\operatorname{curl}, \Omega) \cap H(\operatorname{div}, \Omega)$ equivalent to the initial one. So the bilinear form:

$$
(\psi, \xi) \mapsto \int_{\Omega} \operatorname{curl} \psi \cdot \operatorname{curl} \boldsymbol{\xi} d \boldsymbol{x}
$$

is elliptic on $W$ and, for any $\boldsymbol{u}$ in $L^{2}(\Omega)^{3}$, problem (2.13) has a unique solution $\boldsymbol{\psi}$ in $W$.

The next idea consists in noting that grad $\mu$ belongs to $H_{0}(\operatorname{curl}, \Omega)$ for all $\mu$ in $H(\Omega)$. So, by taking $\boldsymbol{\xi}$ equal to grad $\mu$ and using the Poincaré-Friedrichs inequality, we prove the following inf-sup condition, for a positive constant $\beta$ :

$$
\forall \mu \in H(\Omega), \quad \sup _{\boldsymbol{\xi} \in H_{0}(\operatorname{curl}, \Omega)} \frac{\int_{\Omega} \boldsymbol{\xi} \cdot \operatorname{grad} \mu d \boldsymbol{x}}{\|\boldsymbol{\xi}\|_{H(\operatorname{curl}, \Omega)}} \geq \beta\|\mu\|_{H^{1}(\Omega)} .
$$

This yields the well-posedness of problem (2.10).

Proposition 2.2. For any data $\boldsymbol{u}$ in $L^{2}(\Omega)^{3}$, problem (2.10) has a unique solution $(\boldsymbol{\psi}, \theta)$ in $H_{0}(\mathbf{c u r l}, \Omega) \times H(\Omega)$. Moreover, $\theta$ is equal to zero.

Proof: It remains to prove the last assertion. The idea is to take $\boldsymbol{\xi}$ equal to $\operatorname{grad} \theta$ in the first line of (2.10). This yields

$$
\int_{\Omega}|\operatorname{grad} \theta|^{2} d \boldsymbol{x}=0
$$

So, since $\Omega$ is connected, $\theta$ is a constant and its nullity follows from the boundary conditions that it satisfies on $\Gamma_{0}$.

Remark: It can be noted that problem (2.10) is well-posed even for functions $\boldsymbol{u}$ which do not satisfy (2.7) and that, in this case, the vector field $\boldsymbol{u}$ in (2.10) can be replaced by its projection onto functions satisfying (2.7). We are specially interested in the case where $\boldsymbol{u}$ is not divergence-free but has a small divergence, since in this case curl $\boldsymbol{\psi}$ provides a divergence-free approximation of $\boldsymbol{u}$.

Some further regularity on $\boldsymbol{\psi}$ can be derived from the previous arguments, since it belongs to $H_{0}(\operatorname{curl}, \Omega) \cap H(\operatorname{div}, \Omega)$, see (2.5). Moreover, when $\boldsymbol{u}$ belongs to $H(\operatorname{curl}, \Omega)$, the same properties hold for $\operatorname{curl} \psi$. 


\section{Discretization of the vector potential problem.}

From now on, we assume that $\Omega$ is a three-dimensional bounded connected polyhedron with a Lipschitz-continuous boundary. We also assume that $\Omega$ admits a disjoint decomposition into a finite number of rectangular parallelepipeds, denoted by $\Omega_{k}$ :

$$
\bar{\Omega}=\bigcup_{k=1}^{K} \bar{\Omega}_{k} \quad \text { and } \quad \Omega_{k} \cap \Omega_{k^{\prime}}=\emptyset, \quad 1 \leq k \neq k^{\prime} \leq K .
$$

Note that, as indicated in [21], the extension to more complex subdomains leads to similar results, however it involves very technical arguments that we prefer to avoid in this work. We make the further assumption that the intersection of each $\partial \Omega_{k}$ with $\partial \Omega$, if not empty, is a corner, a whole edge or a whole face of $\Omega_{k}$. For $1 \leq k \leq K$, we denote by $\Gamma_{k, \ell}$, $1 \leq \ell \leq L(k)$, the (open) faces of $\Omega_{k}$ which are not contained in $\partial \Omega$. Let also $\boldsymbol{n}_{k}$ be the unit outward normal vector to $\Omega_{k}$ on $\partial \Omega_{k}$. Note that the decomposition is said to be conforming if the intersection of two different domains $\Omega_{k}$ is either empty or a corner or a whole edge or face of both of them, however we do not make this assumption since it is a priori not necessary for the mortar method.

Let us now introduce the skeleton $\mathcal{S}$ of the decomposition, $\mathcal{S}=\cup_{k=1}^{K} \partial \Omega_{k} \backslash \partial \Omega$. As suggested in [6][7], we choose a disjoint decomposition of this skeleton into mortars $\gamma_{m}$ :

$$
\mathcal{S}=\bigcup_{m=1}^{M} \bar{\gamma}_{m} \quad \text { and } \quad \gamma_{m} \cap \gamma_{m^{\prime}}=\emptyset, \quad 1 \leq m \neq m^{\prime} \leq M,
$$

where each $\gamma_{m}=\Gamma_{k(m), \ell(m)}$ is a whole face of a subdomain $\Omega_{k}$, denoted by $\Omega_{k(m)}$.

To describe the discrete spaces, for each nonnegative integer $n$, we define on each $\Omega_{k}$, resp. $\Gamma_{k, \ell}$, the space $\mathbb{P}_{n}\left(\Omega_{k}\right)$, resp. $\mathbb{P}_{n}\left(\Gamma_{k, \ell}\right)$, of restrictions to $\Omega_{k}$, resp. to $\Gamma_{k, \ell}$, of polynomials with 3 variables, resp. 2 variables (the tangential coordinates on $\Gamma_{k, \ell}$ ), and degree $\leq n$ with respect to each variable. The discretization parameter $\delta$ is then a $K$-tuple of positive integers $\left(N_{1}, \ldots, N_{K}\right)$, with each $N_{k} \geq 2$.

The discrete space corresponding to $H_{0}(\operatorname{curl}, \Omega)$ is defined by analogy with $[2, \S 4]$ : it is the space $\mathbb{C}_{\delta}(\Omega)$ of functions $\boldsymbol{v}_{\delta}$ such that:

- their restrictions $\boldsymbol{v}_{\delta \mid \Omega_{k}}$ to each $\Omega_{k}, 1 \leq k \leq K$, belong to $\mathbb{P}_{N_{k}}\left(\Omega_{k}\right)^{3}$,

- their tangential traces $\boldsymbol{v}_{\delta} \times \boldsymbol{n}$ vanish on $\partial \Omega$,

- the mortar function $\varphi$ being defined on the skeleton by

$$
\boldsymbol{\varphi}_{\mid \gamma_{m}}=\boldsymbol{v}_{\delta \mid \Omega_{k(m)}} \times \boldsymbol{n}_{k(m)}, \quad 1 \leq m \leq M,
$$

the following matching condition holds on each edge $\Gamma_{k, \ell}, 1 \leq k \leq K, 1 \leq \ell \leq L(k)$, which is not a mortar:

$$
\forall \boldsymbol{\chi} \in \mathbb{P}_{N_{k}-2}\left(\Gamma_{k, \ell}\right)^{3}, \quad \int_{\Gamma_{k, \ell}}\left(\boldsymbol{v}_{\delta \mid \Omega_{k}} \times \boldsymbol{n}_{k}+\boldsymbol{\varphi}\right)(\boldsymbol{\tau}) \cdot \boldsymbol{\chi}(\boldsymbol{\tau}) d \boldsymbol{\tau}=0 .
$$

This space is not contained in $H_{0}(\operatorname{curl}, \Omega)$ in the general case. But it provides accurate approximations of functions in this space, see $[2, \S 5]$ for the first results on this subject. 
We also need a mortar approximation of the space $H(\Omega)$, which is not the standard one [7] but is more appropriate for the present problem. It is the space $\mathbb{H}_{\delta}(\Omega)$ of functions $\mu_{\delta}$ such that:

- their restrictions $\mu_{\delta \mid \Omega_{k}}$ to each $\Omega_{k}, 1 \leq k \leq K$, belong to $\mathbb{P}_{N_{k}}\left(\Omega_{k}\right)$,

- the traces of the $\mu_{\delta \mid \Omega_{k}}$ for all $k, 1 \leq k \leq K$, such that $\partial \Omega_{k} \cap \partial \Omega$ has a positive measure, vanish on $\Gamma_{0}$ and are constant on each $\Gamma_{i}, 1 \leq i \leq I$,

- the $\mu_{\delta \mid \Omega_{k}}$ for all $k, 1 \leq k \leq K$, such that $\partial \Omega_{k} \cap \Gamma_{0}$ has a zero measure, belong to $L_{0}^{2}\left(\Omega_{k}\right)$,

- the mortar function $\tilde{\varphi}$ being defined on each $\gamma_{m}, 1 \leq m \leq M$, by $\tilde{\varphi}_{\mid \gamma_{m}}=\operatorname{grad}_{T} \mu_{\delta \mid \Omega_{k(m)}}$ (where $\operatorname{grad}_{T}$ denotes the tangential gradient), the following matching condition holds on each edge $\Gamma_{k, \ell}, 1 \leq k \leq K, 1 \leq \ell \leq L(k)$, which is not a mortar:

$$
\forall \chi \in \mathbb{P}_{N_{k}-2}\left(\Gamma_{k, \ell}\right)^{2}, \quad \int_{\Gamma_{k, \ell}}\left(\operatorname{grad}_{T} \mu_{\delta \mid \Omega_{k}}-\tilde{\boldsymbol{\varphi}}\right)(\boldsymbol{\tau}) \cdot \chi(\boldsymbol{\tau}) d \boldsymbol{\tau}=0
$$

Starting from the standard Gauss-Lobatto formula on ] - 1,1[, we define on each $\Omega_{k}$ and in each direction the nodes $x_{i}^{k}, y_{i}^{k}$ and $z_{i}^{k}$, and the weights $\rho_{i}^{x, k}, \rho_{i}^{y, k}$ and $\rho_{i}^{z, k}$, $0 \leq i \leq N_{k}$, such that the corresponding quadrature formula is exact on $\mathbb{P}_{2 N_{k}-1}\left(\Omega_{k}\right)$. A discrete product is then introduced on each $\Omega_{k}$ by

$$
\left(u_{\delta}, v_{\delta}\right)_{\delta}^{k}=\sum_{i=0}^{N_{k}} \sum_{j=0}^{N_{k}} \sum_{p=0}^{N_{k}} u_{\delta}\left(x_{i}^{k}, y_{j}^{k}, z_{p}^{k}\right) v_{\delta}\left(x_{i}^{k}, y_{j}^{k}, z_{p}^{k}\right) \rho_{i}^{x, k} \rho_{j}^{y, k} \rho_{p}^{z, k} .
$$

This leads to the global discrete product on $\Omega$ :

$$
\left(u_{\delta}, v_{\delta}\right)_{\delta}=\sum_{k=1}^{K}\left(u_{\delta}, v_{\delta}\right)_{\delta}^{k}
$$

which coincides with the scalar product of $L^{2}(\Omega)$ for all functions $u_{\delta}$ annd $v_{\delta}$ such that each product $\left(u_{\delta} v_{\delta}\right)_{\mid \Omega_{k}}, 1 \leq k \leq K$, belongs to $\mathbb{P}_{2 N_{k}-1}\left(\Omega_{k}\right)$. We also define, for $1 \leq k \leq K$, $\mathcal{I}_{\delta}^{k}$ as the Lagrange interpolation operator on all nodes $\left(x_{i}^{k}, y_{j}^{k}, z_{p}^{k}\right), 0 \leq i, j, p \leq N_{k}$, with values in $\mathbb{P}_{N_{k}}\left(\Omega_{k}\right)$, and finally the global operator $\mathcal{I}_{\delta}$ by

$$
\left(\mathcal{I}_{\delta} v\right)_{\mid \Omega_{k}}=\mathcal{I}_{\delta}^{k} v_{\mid \Omega_{k}}, \quad 1 \leq k \leq K .
$$

The discrete problem reads, for any continuous function $\boldsymbol{u}$ on $\bar{\Omega}$ :

Find $\left(\boldsymbol{\psi}_{\delta}, \theta_{\delta}\right)$ in $\mathbb{C}_{\delta}(\Omega) \times \mathbb{H}_{\delta}(\Omega)$ such that

$$
\begin{aligned}
& \forall \boldsymbol{\xi}_{\delta} \in \mathbb{C}_{\delta}(\Omega), \quad\left(\operatorname{curl} \boldsymbol{\psi}_{\delta}, \operatorname{curl} \boldsymbol{\xi}_{\delta}\right)_{\delta}+\left(\boldsymbol{\xi}_{\delta}, \operatorname{grad} \theta_{\delta}\right)_{\delta}=\left(\boldsymbol{u}, \operatorname{curl} \boldsymbol{\xi}_{\delta}\right)_{\delta}, \\
& \forall \mu_{\delta} \in \mathbb{H}_{\delta}(\Omega), \quad\left(\boldsymbol{\psi}_{\delta}, \text { grad } \mu_{\delta}\right)_{\delta}=0 .
\end{aligned}
$$

Remark: It can be observed that adding a constant on each $\Omega_{k}$ to the part $\theta_{\delta}$ of the solution does not modify the problem. Since moreover we are not interested in the approximation of the part $\theta=0$ of the solution of problem $(2.10)$, the second and third $\bullet$ in the 
definition of $\mathbb{H}_{\delta}(\Omega)$ can without any change be replaced for instance by

- the trace of $\mu_{\delta}$ on $\partial \Omega$ is such that $\operatorname{grad}_{T} \mu_{\delta}$ vanishes on $\partial \Omega$,

- the $\mu_{\delta \mid \Omega_{k}}$ for all $k, 1 \leq k \leq K$, belong to $L_{0}^{2}\left(\Omega_{k}\right)$.

This is only a simpler way of "fixing the constants".

We first introduce the discrete kernel

$$
\mathbb{W}_{\delta}(\Omega)=\left\{\boldsymbol{\xi}_{\delta} \in \mathbb{C}_{\delta}(\Omega) ; \forall \mu_{\delta} \in \mathbb{H}_{\delta}(\Omega),\left(\boldsymbol{\xi}_{\delta}, \operatorname{grad} \mu_{\delta}\right)_{\delta}=0\right\}
$$

So, for any solution $\left(\boldsymbol{\psi}_{\delta}, \theta_{\delta}\right)$ of problem $(3.7)$, the function $\boldsymbol{\psi}_{\delta}$ is a solution of the reduced problem:

Find $\boldsymbol{\psi}_{\delta}$ in $\mathbb{W}_{\delta}(\Omega)$ such that

$$
\forall \boldsymbol{\xi}_{\delta} \in \mathbb{W}_{\delta}(\Omega), \quad\left(\operatorname{curl} \boldsymbol{\psi}_{\delta}, \operatorname{curl} \boldsymbol{\xi}_{\delta}\right)_{\delta}=\left(\boldsymbol{u}, \operatorname{curl} \boldsymbol{\xi}_{\delta}\right)_{\delta}
$$

We first check that this equation is well-posed.

Lemma 3.1. For any function $\boldsymbol{u}$ continuous on $\bar{\Omega}$, problem (3.9) has a unique solution $\psi_{\delta}$ in $\mathbb{W}_{\delta}(\Omega)$.

Proof: Equation (3.9) results into a square linear system, so that the existence of a solution follows from its uniqueness. Thus, we take $\boldsymbol{u}$ equal to zero, which yields

$$
\left(\operatorname{curl} \psi_{\delta}, \operatorname{curl} \psi_{\delta}\right)_{\delta}=0
$$

Then, each $\left(\operatorname{curl} \boldsymbol{\psi}_{\delta}\right)_{\mid \Omega_{k}}, 1 \leq k \leq K$, belongs to $\mathbb{P}_{N_{k}}\left(\Omega_{k}\right)^{3}$ and vanishes on the $\left(N_{k}+1\right)^{3}$ distinct points of a tensorized grid, hence is zero. As a consequence, there exists a function $\chi_{\delta}^{k}$, defined up to an additive constant, such that $\psi_{\delta \mid \Omega_{k}}$ is equal to grad $\chi_{\delta}^{k}$. It is readily checked that $\chi_{\delta}^{k}$ belongs to $\mathbb{P}_{N_{k}}\left(\Omega_{k}\right)$. Moreover, since $\boldsymbol{\psi}_{\delta} \times \boldsymbol{n}$ vanishes on $\partial \Omega$, the same property holds for $\operatorname{grad}_{T} \chi_{\delta}$, where $\chi_{\delta}$ is defined by $\chi_{\delta \mid \Omega_{k}}=\chi_{\delta}^{k}$. So the constant on all $\Omega_{k}$ can be chosen such that $\chi_{\delta \mid \Omega_{k}}$ for all $k$ such that $\partial \Omega_{k} \cap \partial \Omega$ has a positive measure, has a null trace on $\Gamma_{0}$ and a constant trace on each $\Gamma_{i}, 1 \leq i \leq I$, and belong to $L_{0}^{2}\left(\Omega_{k}\right)$ otherwise. Finally, it is readily checked that, on each face of $\Omega_{k}, \operatorname{grad}_{T} \chi_{\delta \mid \Omega_{k}}$ is equal to $\psi_{\delta \mid \Omega_{k}}$ up to the sign, so that the matching conditions on $\boldsymbol{\psi}_{\delta}$ on all $\Gamma_{k, \ell}$ that are not mortars yield the desired conditions on $\chi_{\delta}$. Thus $\chi_{\delta}$ belongs to $\mathbb{H}_{\delta}(\Omega)$. The nullity of $\boldsymbol{\psi}_{\delta}$ is then derived from the orthogonality condition in $\mathbb{W}_{\delta}(\Omega)$, see $(3.8)$.

Lemma 3.2. The space of functions $\mu_{\delta}$ in $\mathbb{H}_{\delta}(\Omega)$ such that

$$
\forall \boldsymbol{\xi}_{\delta} \in \mathbb{C}_{\delta}(\Omega), \quad\left(\boldsymbol{\xi}_{\delta}, \operatorname{grad} \mu_{\delta}\right)_{\delta}=0,
$$

is reduced to $\{0\}$.

Proof: It is readily checked that, for any $\mu_{\delta}$ in $\mathbb{H}_{\delta}(\Omega)$, grad $\mu_{\delta}$ belongs to $\mathbb{C}_{\delta}(\Omega)$. So taking $\boldsymbol{\xi}_{\delta}$ equal to grad $\mu_{\delta}$ in (3.10) yields that each $\mu_{\delta \mid \Omega_{k}}, 1 \leq k \leq K$, is constant, hence zero due to the definition of $\mathbb{H}_{\delta}(\Omega)$. 
Lemma 3.2 states that there is no spurious modes for the Lagrange multiplier in problem (3.7) or equivalently that the following inf-sup condition holds: There exists a constant $\beta_{\delta}$ possibly depending on $\delta$ such that

$$
\forall \mu_{\delta} \in \mathbb{H}_{\delta}(\Omega), \quad \sup _{\boldsymbol{\xi}_{\delta} \in \mathbb{C}_{\delta}(\Omega)} \frac{\left(\boldsymbol{\xi}_{\delta}, \operatorname{grad} \mu_{\delta}\right)_{\delta}}{\left\|\boldsymbol{\xi}_{\delta}\right\|_{H\left(\operatorname{curl}, \cup \Omega_{k}\right)}} \geq \beta_{\delta}\left\|\mu_{\delta}\right\|_{H^{1}\left(\cup \Omega_{k}\right)}
$$

where the broken norms $\|\cdot\|_{H\left(\operatorname{curl}, \cup \Omega_{k}\right)}$ and $\|\cdot\|_{H^{1}\left(\cup \Omega_{k}\right)}$ are defined in an obvious way by

$$
\begin{gathered}
\left\|\boldsymbol{\xi}_{\delta}\right\|_{H\left(\mathrm{curl}, \cup \Omega_{k}\right)}=\left(\sum_{k=1}^{K}\left\|\boldsymbol{\xi}_{\delta \mid \Omega_{k}}\right\|_{H\left(\operatorname{curl}, \Omega_{k}\right)}^{2}\right)^{\frac{1}{2}} \\
\left\|\mu_{\delta}\right\|_{H^{1}\left(\cup \Omega_{k}\right)}=\left(\sum_{k=1}^{K}\left\|\mu_{\delta \mid \Omega_{k}}\right\|_{H^{1}\left(\Omega_{k}\right)}^{2}\right)^{\frac{1}{2}} .
\end{gathered}
$$

This leads to the following result.

Proposition 3.3. For any function $\boldsymbol{u}$ continuous on $\bar{\Omega}$, problem (3.7) has a unique solution $\left(\boldsymbol{\psi}_{\delta}, \theta_{\delta}\right)$ in $\mathbb{C}_{\delta}(\Omega) \times \mathbb{H}_{\delta}(\Omega)$. Moreover, $\theta_{\delta}$ is equal to zero.

Proof: Since problem (3.7) is equivalent to a square linear system, the existence and uniqueness of the solution $\left(\boldsymbol{\psi}_{\delta}, \theta_{\delta}\right)$ is derived from Lemma 3.1, combined with (3.11). Next, using the same argument as in the proof of Lemma 3.2, we take $\boldsymbol{\xi}_{\delta}$ equal to grad $\theta_{\delta}$, which yields that $\theta_{\delta}$ vanishes on $\Omega$. 


\section{Error analysis.}

We are interested in evaluating the quantity $\left\|\boldsymbol{u}-\operatorname{curl} \boldsymbol{\psi}_{\delta}\right\|_{L^{2}(\Omega)^{3}}$, where $\left(\boldsymbol{\psi}_{\delta}, 0\right)$ is the solution of problem (3.7). From the first line in (2.8), we observe that

$$
\forall \boldsymbol{\xi}_{\delta} \in \mathbb{C}_{\delta}(\Omega), \quad \sum_{k=1}^{K} \int_{\Omega_{k}} \operatorname{curl} \boldsymbol{\psi} \cdot \operatorname{curl} \boldsymbol{\xi}_{\delta} d \boldsymbol{x}=\sum_{k=1}^{K} \int_{\Omega_{k}} \boldsymbol{u} \cdot \operatorname{curl} \boldsymbol{\xi}_{\delta} d \boldsymbol{x}
$$

so that no consistency error due to the nonconformity of the method appears in this line. We recall from the standard property of the Gauss-Lobatto formula [5, Rem. 13.3] that

$$
\forall z_{\delta} \in \mathbb{P}_{N_{k}}\left(\Omega_{k}\right), \quad\left\|z_{\delta}\right\|_{L^{2}\left(\Omega_{k}\right)}^{2} \leq\left(z_{\delta}, z_{\delta}\right)_{\delta}^{k} \leq 3^{3}\left\|z_{\delta}\right\|_{L^{2}\left(\Omega_{k}\right)}^{2}
$$

So we have, for all $\boldsymbol{\xi}_{\delta}$ in $\mathbb{C}_{\delta}(\Omega)$,

$$
\left\|\operatorname{curl} \psi_{\delta}-\operatorname{curl} \xi_{\delta}\right\|_{L^{2}(\Omega)^{3}}^{2} \leq\left(\operatorname{curl} \psi_{\delta}-\operatorname{curl} \boldsymbol{\xi}_{\delta}, \operatorname{curl} \psi_{\delta}-\operatorname{curl} \boldsymbol{\xi}_{\delta}\right)_{\delta} .
$$

Using (3.7) (with $\theta_{\delta}=0$ ) yields

$$
\begin{aligned}
\left\|\operatorname{curl} \boldsymbol{\psi}_{\delta}-\operatorname{curl} \boldsymbol{\xi}_{\delta}\right\|_{L^{2}(\Omega)^{3}}^{2} \leq\left(\boldsymbol{u}-\operatorname{curl} \boldsymbol{\xi}_{\delta},\right. & \left.\operatorname{curl} \boldsymbol{\psi}_{\delta}-\operatorname{curl} \boldsymbol{\xi}_{\delta}\right)_{\delta} \\
& =\left(\mathcal{I}_{\delta} \boldsymbol{u}-\operatorname{curl} \boldsymbol{\xi}_{\delta}, \operatorname{curl} \boldsymbol{\psi}_{\delta}-\operatorname{curl} \boldsymbol{\xi}_{\delta}\right)_{\delta},
\end{aligned}
$$

where the operator $\mathcal{I}_{\delta}$ is introduced in (3.6). Using once more (4.2) thus gives

$$
\left\|\operatorname{curl} \boldsymbol{\psi}_{\delta}-\operatorname{curl} \boldsymbol{\xi}_{\delta}\right\|_{L^{2}(\Omega)^{3}} \leq 3^{3}\left\|\mathcal{I}_{\delta} \boldsymbol{u}-\operatorname{curl} \boldsymbol{\xi}_{\delta}\right\|_{L^{2}(\Omega)^{3}}
$$

whence, thanks to triangle inequalities,

$$
\left\|\boldsymbol{u}-\operatorname{curl} \boldsymbol{\psi}_{\delta}\right\|_{L^{2}(\Omega)^{3}} \leq c\left(\inf _{\boldsymbol{\xi}_{\delta} \in \mathbb{C}_{\delta}(\Omega)}\left\|\boldsymbol{u}-\operatorname{curl} \boldsymbol{\xi}_{\delta}\right\|_{L^{2}(\Omega)^{3}}+\left\|\boldsymbol{u}-\mathcal{I}_{\delta} \boldsymbol{u}\right\|_{L^{2}(\Omega)^{3}}\right)
$$

The approximation properties of the operator $\mathcal{I}_{\delta}$ are stated in [5, Thm 14.2]: For any function $v$ in $H^{s}\left(\Omega_{k}\right), s>\frac{3}{2}$,

$$
\left\|v-\mathcal{I}_{\delta}^{k} v\right\|_{L^{2}\left(\Omega_{k}\right)} \leq c N_{k}^{-s}\|v\|_{H^{s}\left(\Omega_{k}\right)} .
$$

In order to estimate the distance of $\boldsymbol{u}$ to the curl of $\mathbb{C}_{\delta}(\Omega)$, we recall the following result from [2, Lemma 5.2].

Lemma 4.1. Assume that the function $\boldsymbol{u}$ satisfies (2.7) and is such that each $\boldsymbol{u}_{\mid \Omega_{k}}$, $1 \leq k \leq K$, belongs to $H^{s_{k}}\left(\Omega_{k}\right)^{3}, s_{k} \geq \frac{3}{2}$. If the decomposition is conforming and if moreover for each mortar $\gamma^{m}, 1 \leq m \leq M$, which is a face of both $\Omega_{k(m)}$ and $\Omega_{k}, N_{k(m)}$ is $\geq N_{k}$, there exists a constant $c$ independent of $\delta$ such that

$$
\inf _{\boldsymbol{\xi}_{\delta} \in \mathbb{C}_{\delta}(\Omega)}\left\|\boldsymbol{u}-\operatorname{curl} \boldsymbol{\xi}_{\delta}\right\|_{L^{2}(\Omega)^{3}} \leq c \sum_{k=1}^{K} N_{k}^{-s_{k}}\|\boldsymbol{u}\|_{H^{s_{k}}\left(\Omega_{k}\right)^{3}}
$$


In the case of non conforming decompositions, we refer to [10, Prop. 11] for the next result.

Lemma. 4.2. Assume that the function $\boldsymbol{\psi}$ is such that each $\boldsymbol{\psi}_{\mid \Omega_{k}}, 1 \leq k \leq K$, belongs to $H^{s_{k}+1}\left(\Omega_{k}\right)^{3}, s_{k}>\frac{3}{2}$. If the ratios $N_{k} / N_{k^{\prime}}$ for all subdomains $\Omega_{k}$ and $\Omega_{k^{\prime}}$ such that $\partial \Omega_{k} \cap$ $\partial \Omega_{k^{\prime}}$ is not empty, are bounded independently of $\delta$, there exists a constant $c$ independent of $\delta$ such that

$$
\inf _{\boldsymbol{\xi}_{\delta} \in \mathbb{C}_{\delta}(\Omega)}\left\|\operatorname{curl} \boldsymbol{\psi}-\operatorname{curl} \boldsymbol{\xi}_{\delta}\right\|_{L^{2}(\Omega)^{3}} \leq c \sum_{k=1}^{K} N_{k}^{\frac{1}{4}-s_{k}}\|\boldsymbol{\psi}\|_{H^{s_{k}+1}\left(\Omega_{k}\right)^{3}}
$$

Estimate (4.5) proves the optimal approximation properties of the space $\mathbb{C}_{\delta}(\Omega)$ in the case of a conforming decomposition (indeed taking $N_{k(m)} \geq N_{k}$ is not restrictive in this case). Estimte (4.6) is no longer optimal but deals with the general nonconforming decomposition which is much more complex. Moreover the lack of optimality is of order $\left(\max _{1 \leq k \leq K} N_{k}\right)^{\frac{1}{4}}$, so is not too high, and there also the fact that all ratios $N_{k} / N_{k^{\prime}}$ are bounded for adjacent domains $\Omega_{k}$ and $\Omega_{k^{\prime}}$ is not restrictive.

Theorem 4.3. Assume that the function $\boldsymbol{u}$ satisfies (2.7), that the fonction $\boldsymbol{\psi}$ satisfies (2.8) and is such that each $\boldsymbol{\psi}_{\mid \Omega_{k}}, 1 \leq k \leq K$, belongs to $H^{s_{k}+1}\left(\Omega_{k}\right)^{3}, s_{k}>\frac{3}{2}$. Then, if the ratios $N_{k} / N_{k^{\prime}}$ for all subdomains $\Omega_{k}$ and $\Omega_{k^{\prime}}$ such that $\partial \Omega_{k} \cap \partial \Omega_{k^{\prime}}$ is not empty, are bounded independently of $\delta$, the following error estimate holds between this function $\boldsymbol{u}$ and the function curl $\boldsymbol{\psi}_{\delta}$ issued from problem (3.7):

$$
\left\|\boldsymbol{u}-\operatorname{curl} \boldsymbol{\psi}_{\delta}\right\|_{L^{2}(\Omega)^{3}} \leq c \sum_{k=1}^{K} N_{k}^{\frac{1}{4}-s_{k}}\|\boldsymbol{\psi}\|_{H^{s_{k}+1}\left(\Omega_{k}\right)^{3}} .
$$

Moreover, if the decomposition is conforming and if, for each mortar $\gamma^{m}, 1 \leq m \leq M$, which is a face of both $\Omega_{k(m)}$ and $\Omega_{k}, N_{k(m)}$ is $\geq N_{k}$, this estimate can be improved as follows

$$
\left\|\boldsymbol{u}-\operatorname{curl} \boldsymbol{\psi}_{\delta}\right\|_{L^{2}(\Omega)^{3}} \leq c \sum_{k=1}^{K} N_{k}^{-s_{k}}\|\boldsymbol{u}\|_{H^{s_{k}\left(\Omega_{k}\right)^{3}}} .
$$

Estimate (4.8) is fully optimal and only involve the regularity of the data $\boldsymbol{u}$. When the functions $\boldsymbol{u}$ does not satisfy (2.7), exactly the same estimate holds either with $\boldsymbol{u}$ replaced by its projection onto the space of functions satisfying (2.7) or with a further regularity assumption on the function $q$ which appears in (2.6). Moreover, note that the restriction $s_{k}>\frac{3}{2}$ comes from the use of the quadrature formula, however it does not seem too restrictive. On the other hand, even if estimate (4.7) is not fully optimal, numerical experiments show the good convergence of the discretization even for nonconforming decompositions. 


\section{Numerical algorithms and experiments.}

We now explain how to translate the discrete problem (3.7) into a square linear system, and we propose algorithms for solving this sytem. Since this is very similar to [2, $\S 7]$, we only give a brief description of these algorithms which rely on the following idea, due to [4]: The matching conditions through the skeleton can be handled via the introduction of a further Lagrange multiplier.

Let now $\overline{\mathbb{C}}_{\delta}(\Omega)$ be the space of functions $\boldsymbol{v}_{\delta}$ such that

- their restrictions $\boldsymbol{v}_{\delta \mid \Omega_{k}}$ to each $\Omega_{k}, 1 \leq k \leq K$, belong to $\mathbb{P}_{N_{k}}\left(\Omega_{k}\right)^{3}$,

- their tangential traces $\boldsymbol{v}_{\delta} \times \boldsymbol{n}$ vanish on $\partial \Omega$,

and $\overline{\mathbb{H}}_{\delta}(\Omega)$ the space of functions $\mu_{\delta}$ such that

- their restrictions $\mu_{\delta \mid \Omega_{k}}$ to each $\Omega_{k}, 1 \leq k \leq K$, belong to $\mathbb{P}_{N_{k}}\left(\Omega_{k}\right) \cap L_{0}^{2}\left(\Omega_{k}\right)$,

- the tangential gradient of the traces of the $\mu_{\delta \mid \Omega_{k}}$ for all $k, 1 \leq k \leq K$, such that $\partial \Omega_{k} \cap \partial \Omega$ has a positive measure, vanish.

We also need the space $\mathbb{L}_{\delta}(\mathcal{S})$ of functions $\nu_{\delta}$ in $L^{2}(\mathcal{S})$ such that their restrictions to each $\Gamma_{k, \ell}$ which is not a mortar belong to $\mathbb{P}_{N_{k}-2}\left(\Gamma_{k, \ell}\right)$. The set of indices $(k, \ell), 1 \leq k \leq K$, $1 \leq \ell \leq L(k)$, such that $\Gamma_{k, \ell}$ is not a mortar is denoted by $\mathcal{K}$.

We thus consider the modified problem:

$$
\begin{aligned}
& \text { Find }\left(\boldsymbol{\psi}_{\delta}, \boldsymbol{\pi}_{\delta}, \theta_{\delta}, \boldsymbol{\sigma}_{\delta}\right) \text { in } \overline{\mathbb{C}}_{\delta}(\Omega) \times \mathbb{L}_{\delta}(\mathcal{S})^{3} \times \overline{\mathbb{H}}_{\delta}(\Omega) \times \mathbb{L}_{\delta}(\mathcal{S})^{2} \text { such that } \\
& \forall \boldsymbol{\xi}_{\delta} \in \overline{\mathbb{C}}_{\delta}(\Omega), \quad\left(\operatorname{curl} \boldsymbol{\psi}_{\delta}, \operatorname{curl} \boldsymbol{\xi}_{\delta}\right)_{\delta}+\left(\boldsymbol{\xi}_{\delta}, \operatorname{grad} \theta_{\delta}\right)_{\delta} \\
& +c_{\delta}\left(\boldsymbol{\xi}_{\delta}, \boldsymbol{\pi}_{\delta}\right)=\left(\boldsymbol{u}, \operatorname{curl} \boldsymbol{\xi}_{\delta}\right)_{\delta}, \\
& \forall \boldsymbol{\rho}_{\delta} \in \mathbb{L}_{\delta}(\mathcal{S})^{3}, \quad c_{\delta}\left(\boldsymbol{\psi}_{\delta}, \boldsymbol{\rho}_{\delta}\right)=0, \\
& \forall \mu_{\delta} \in \overline{\mathbb{H}}_{\delta}(\Omega), \quad\left(\boldsymbol{\psi}_{\delta}, \operatorname{grad} \mu_{\delta}\right)_{\delta}+h_{\delta}\left(\mu_{\delta}, \boldsymbol{\sigma}_{\delta}\right)=0, \\
& \forall \chi_{\delta} \in \mathbb{L}_{\delta}(\mathcal{S})^{2}, \quad h_{\delta}\left(\theta_{\delta}, \chi_{\delta}\right)=0 \text {, }
\end{aligned}
$$

where the bilinear forms $c_{\delta}(\cdot, \cdot)$ and $h_{\delta}(\cdot, \cdot)$ are defined by

$$
\begin{aligned}
& c_{\delta}\left(\boldsymbol{\vartheta}_{\delta}, \boldsymbol{\rho}_{\delta}\right)=\sum_{(k, \ell) \in \mathcal{K}} \int_{\Gamma_{k, \ell}}\left(\boldsymbol{\vartheta}_{\delta \mid \Omega_{k}} \times \boldsymbol{n}_{k}+\boldsymbol{\varphi}\left(\boldsymbol{\vartheta}_{\delta}\right)\right)(\boldsymbol{\tau}) \cdot \boldsymbol{\rho}_{\delta}(\boldsymbol{\tau}) d \boldsymbol{\tau} \\
& h_{\delta}\left(\mu_{\delta}, \boldsymbol{\chi}_{\delta}\right)=\sum_{(k, \ell) \in \mathcal{K}} \int_{\Gamma_{k, \ell}}\left(\operatorname{grad}_{T} \mu_{\delta} \mid \Omega_{k}-\tilde{\boldsymbol{\varphi}}\left(\mu_{\delta}\right)\right)(\boldsymbol{\tau}) \cdot \chi_{\delta}(\boldsymbol{\tau}) d \boldsymbol{\tau}
\end{aligned}
$$

with obvious definitions of the mortar functions $\varphi(\cdot)$ and $\tilde{\varphi}(\cdot)$, see (3.3) and (3.4). Clearly, for any solution $\left(\boldsymbol{\psi}_{\delta}, \boldsymbol{\pi}_{\delta}, \theta_{\delta}, \boldsymbol{\sigma}_{\delta}\right)$ of problem $(5.1),\left(\boldsymbol{\psi}_{\delta}, \theta_{\delta}\right)$ is a solution of problem (3.7).

Problem (5.1) is equivalent to the following linear system

$$
\left(\begin{array}{cccc}
D & E & G & 0 \\
E^{T} & 0 & 0 & 0 \\
G^{T} & 0 & 0 & H \\
0 & 0 & H^{T} & 0
\end{array}\right)\left(\begin{array}{c}
\Psi \\
\Pi \\
\Theta \\
\Sigma
\end{array}\right)=\left(\begin{array}{c}
A U \\
0 \\
0 \\
0
\end{array}\right)
$$


where the vector $U$ is made of the values of $\boldsymbol{u}$ at all nodes $\left(x_{i}^{k}, y_{j}^{k}, z_{p}^{k}\right), 0 \leq i, j, p \leq N_{k}$, $1 \leq k \leq K$, and the matrix $A$ is fully diagonal, its diagonal terms being the $\rho_{i}^{x, k} \rho_{j}^{y, k} \rho_{p}^{z, k}$. The main unknown is the vector $\Psi$ of the values of $\boldsymbol{\psi}_{\delta}$ at all nodes $\left(x_{i}^{k}, y_{j}^{k}, z_{p}^{k}\right), 0 \leq i, j, p \leq$ $N_{k}, 1 \leq k \leq K$. The matrix $G$ is block-diagonal, with one block per each subdomain $\Omega_{k}$, and the matrix $D$ is symmetric, whence the symmetry of the global system. The number of non-zero coefficients in $E$ and $H$ is very small.

System (5.2) is solved via a stabilized bi-gradient algorithm. We refer to [19, §5.5] for more details on this procedure in a slightly different framework.

The numerical experiments that we present are aimed to prove the convergence of the method. The domain $\Omega$ is the cube $]-1,1\left[^{3}\right.$, with the following non-conforming decomposition

$$
\left.\Omega_{1}=\right]-1,1[2 \times]-1,0\left[, \quad \Omega_{2}=\right]-1,0[\times]-1,1[\times] 0,1\left[, \quad \Omega_{3}=\right] 0,1[\times]-1,1[\times] 0,1[.
$$

We have chosen as mortars the three faces

$$
\left.\gamma_{1}=\right]-1,0[\times]-1,1\left[\times\{0\}, \quad \gamma_{2}=\right] 0,1[\times]-1,1\left[\times\{0\}, \quad \gamma_{3}=\{0\} \times\right]-1,1[\times] 0,1[.
$$

We work with the smooth data $\boldsymbol{u}$ given by

$$
\begin{aligned}
\boldsymbol{u}(x, y, z)=\left(\chi(x) \chi^{\prime}(y) \chi^{\prime}(z), 4 \chi^{\prime}(x) \chi(y) \chi^{\prime}(z)\right. & \left.-5 \chi^{\prime}(x) \chi^{\prime}(y) \chi(z)\right) \\
\text { with } \quad \chi(t) & =\sinh (2) \sinh (t)-\sinh (1) \sinh (2 t)
\end{aligned}
$$

which obviously satisfies the conditions (2.7).

Table 1 presents the maximum of the three components of the error $\boldsymbol{u}-\operatorname{curl} \boldsymbol{\psi}_{\delta}$ at the Gauss-Lobatto nodes contained in the $\bar{\Omega}_{k}, 1 \leq k \leq 3$, and in the $\gamma_{m}, 1 \leq m \leq 3$, for the discretization parameter $\delta=\left(N_{1}, N_{2}, N_{3}\right)$ given by

$$
N_{1}=12, \quad N_{2}=10, \quad N_{3}=8
$$

Table 2 presents the same quantities for the discretization parameter given by

$$
N_{1}=16, \quad N_{2}=14, \quad N_{3}=12 .
$$

In this table, the symbol $\sharp$ means the zero machine value.

\begin{tabular}{|l|c|c|c|c|c|c|}
\hline & $\Omega_{1}$ & $\Omega_{2}$ & $\Omega_{3}$ & $\gamma_{1}$ & $\gamma_{2}$ & $\gamma_{3}$ \\
\hline$x$-comp. & $2.18 .10^{-6}$ & $4.24 .10^{-6}$ & $4.21 .10^{-6}$ & $2.18 .10^{-6}$ & $2.08 .10^{-6}$ & $4.21 .10^{-6}$ \\
$y$-comp. & $2.37 .10^{-5}$ & $2.34 .10^{-5}$ & $2.37 .10^{-5}$ & $6.96 .10^{-6}$ & $2.37 .10^{-5}$ & $2.34 .10^{-5}$ \\
$z$-comp. & $5.66 .10^{-6}$ & $2.33 .10^{-6}$ & $4.32 .10^{-6}$ & $5.59 .10^{-7}$ & $4.32 .10^{-6}$ & $1.81 .10^{-6}$ \\
\hline
\end{tabular}

TABLE 1: The maximum of the error for the discretization parameter given in (5.4) 


\begin{tabular}{|l|c|c|c|c|c|c|}
\hline & $\Omega_{1}$ & $\Omega_{2}$ & $\Omega_{3}$ & $\gamma_{1}$ & $\gamma_{2}$ & $\gamma_{3}$ \\
\hline$x$-comp. & $2.03 .10^{-13}$ & $3.18 .10^{-14}$ & $2.03 .10^{-13}$ & $3.18 .10^{-14}$ & $2.03 .10^{-13}$ & $\sharp$ \\
$y$-comp. & $6.03 .10^{-11}$ & $6.02 .10^{-11}$ & $6.03 .10^{-11}$ & $2.68 .10^{-13}$ & $6.03 .10^{-11}$ & $6.02 .10^{-11}$ \\
$z$-comp. & $\sharp$ & $9.68 .10^{-13}$ & $9.68 .10^{-13}$ & $\sharp$ & $\sharp$ & $9.68 .10^{-13}$ \\
\hline
\end{tabular}

TABLE 2: The maximum of the error for the discretization parameter given in (5.5)

The curves of isovalues of the three components of the discrete solution curl $\psi_{\delta}$ in the plane $y=\frac{1}{2}$ and for the discretization parameter given in (5.5) are presented in Figure 1 (their analogues for the parameter in (5.4) are completely similar).

$\mathbf{y}=\mathbf{0 . 5}$

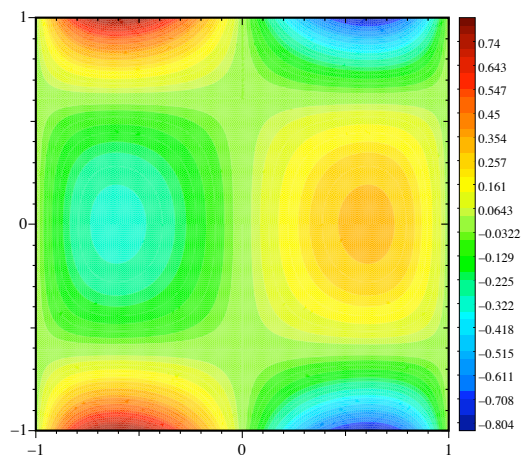

$\mathbf{y}=\mathbf{0 . 5}$

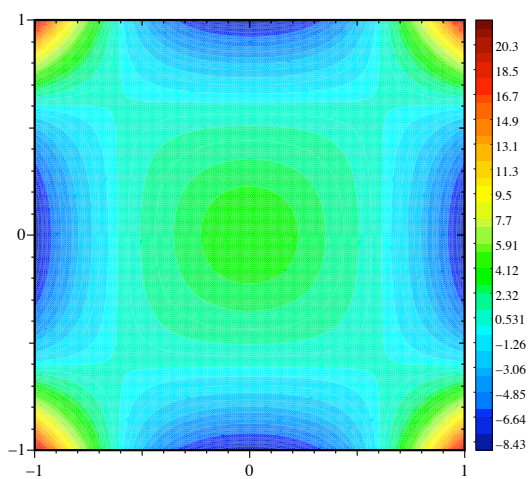

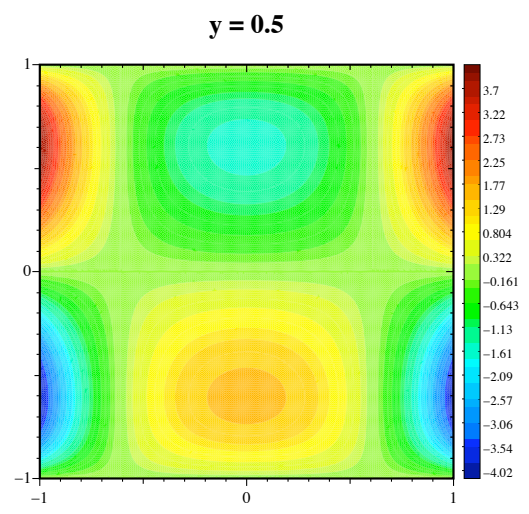

Figure 1: The isovalues of the discrete solution $\operatorname{curl} \boldsymbol{\psi}_{\delta}$

It can be noted that the error, which is already of order $10^{-6}$ in Table 1 , is still reduced in Table 2, so that the convergence is really of spectral type. Moreover the continuity of the tangential trace of $\psi_{\delta}$ through the $\Gamma_{k, \ell}$ is correctly enforced, despite the nonconformity of the method. 


\section{References}

[1] C. Amrouche, C. Bernardi, M. Dauge, V. Girault - Vector potentials in three-dimensional non-smooth domains, Math. Meth. in the Appl. Sc. 21 (1998), 823-864.

[2] M. Azaïez, F. Ben Belgacem, C. Bernardi - The mortar spectral element method in domains of operators. Part I: The divergence operator and Darcy's equations, IMA J. Numer. Anal. 26 (2006), 131-154.

[3] A. Ben Abdallah, F. Ben Belgacem, Y. Maday, F. Rapetti - Mortaring the two-dimensional edge finite elements for the discretization of some electromagnetic models, Math. Models and Methods in Applied Sciences 14 (2004), 1635-1656.

[4] F. Ben Belgacem - The Mortar finite element method with Lagrangian multiplier, Numer. Math. 84 (1999), 173-197.

[5] C. Bernardi, Y. Maday - Spectral Methods, in the Handbook of Numerical Analysis, Vol. V, P.G. Ciarlet \& J.-L. Lions eds., North-Holland (1997).

[6] C. Bernardi, Y. Maday, A.T. Patera - A new nonconforming approach to domain decomposition: the mortar element method, Collège de France Seminar XI, H. Brezis \& J.-L. Lions eds., Pitman (1994), 13-51.

[7] C. Bernardi, Y. Maday, A.T. Patera - Domain decomposition by the mortar element method, in Asymptotic and Numerical Methods for Partial Differential Equations with Critical Parameters, H.G. Kaper \& M. Garbey eds., N.A.T.O. ASI Series C 384 (1993), 269-286.

[8] C. Bernardi, Y. Maday, F. Rapetti - Basics and some applications of the mortar element method, GAMM - Gesellschaft für Angewandte Mathematik und Mechanik 28 (2005), 97-123.

[9] A. Bossavit - Electromagnétisme en vue de la modélisation, "Mathématiques et Applications" 14, Springer-Verlag (1993).

[10] T.Z. Boulmezaoud, M. El Rhabi - A mortar spectral element method for 3D Maxwell's equations, IMA J. Numer. Anal. 25 (2005), 577-610.

[11] A. Buffa, P. Ciarlet Jr. - On traces for functional spaces related to Maxwell's equations. Part I: An integration by parts formula in Lipschitz polyhedra, Math. Meth. Appl. Sci. 24 (2001), 9-30.

[12] A. Buffa, P. Ciarlet Jr. - On traces for functional spaces related to Maxwell's equations. Part II: Hodge decompositions on the boundary of Lipschitz polyhedra and applications, Math. Meth. Appl. Sci. 24 (2001), 31-48.

[13] A. Buffa, M. Costabel, D. Sheen - On traces for $H($ curl, $\Omega)$ in Lipschitz domains, J. Math. Anal. Appl. 276 (2002), 845-867.

[14] A. Buffa, Y. Maday, F. Rapetti - A sliding mesh-mortar method for a two dimensional eddy currents model of electric engines, Modél. Math. et Anal. Numér. 35 (2001), 191-228. 
[15] M. Costabel - A remark on the regularity of solutions of Maxwell's equations on Lipschitz domains, Math. Meth. in Appl. Sc. 12 (1990), 365-368.

[16] M. Costabel, M. Dauge - Espaces fonctionnels Maxwell: Les gentils, les méchants et les singularités, Web Publications (1998), http://perso.univ-rennes1.fr/monique.dauge.

[17] M. Costabel, M. Dauge - Computation of resonance frequencies for Maxwell equations in non smooth domains, in Topics in Computational Wave Propagation, M. Ainsworth, P. Davies, D. Duncan, P. Martin and B. Rynne eds., Springer (2004), 125-161.

[18] M. Dauge - Neumann and mixed problems on curvilinear polyhedra, Integr. Equat. Oper. Th. 15 (1992), 227-261.

[19] M. El Rhabi - Analyse numérique et discrétisation par éléments spectraux avec joints des équatons tridimensionnelles de l'électromagnétisme, Thesis, Université Pierre et Marie Curie, Paris (2002).

[20] V. Girault, P.-A. Raviart — Finite Element Methods for Navier-Stokes Equations, Theory and Algorithms, Springer-Verlag (1986).

[21] Y. Maday, E.M. Rønquist - Optimal error analysis of spectral methods with emphasis on nonconstant coefficients and deformed geometries, Comp. Methods in Applied Mech. and Engrg. 80 (1990), 91-115.

[22] F. Rapetti - Approximation des équations de la magnétodynamique en domaine tournant par la méthode des éléments avec joints, Thesis, Université Pierre et Marie Curie, Paris (2000).

[23] L. Tartar - On the characterization of traces of a Sobolev space used for Maxwell's equation, in the Proceedings of a meeting held in honor of M. Artola, A.-Y. Le Roux ed., Bordeaux (1997). 